\title{
Importance of Neutrophil/Lymphocyte Ratio in Prediction of PSA Recurrence after Radical Prostatectomy
}

\author{
Eymen Gazel' ${ }^{1}$, Sedat Tastemur'1, Onur Acikgoz ${ }^{1}$, Metin Yigman', Erkan \\ Olcucuoglu $^{1}$, Ahmet Camtosun ${ }^{1}$, Cavit Ceylan ${ }^{1}$, Can Ates ${ }^{2}$
}

\begin{abstract}
Background: The aim of this study was to research the importance of the neutrophil to lymphocyte ratio (NLR) in prediction of PSA recurrence after radical prostatectomy, which has not been reported so far. Materials and Methods: The data of 175 patients who were diagnosed with localised prostate cancer and underwent retropubic radical prostatectomy was retrospectively examined. Patient pre-operative hemogram parameters of neutrophil count, lymphocyte count and NLR were assessed. The patients whose PSAs were too low to measure after radical prostatectomy in their follow-ups, and then had PSAs of $0,2 \mathrm{ng} / \mathrm{mL}$ were considered as patients with PSA recurrence. Patients with recurrence made up Group A and patients without recurrence made up Group B. Results: In terms of the power of NLR value in distinguishing recurrence, the area under OCC was statistically significant $(p<0.001)$.The value of 2.494 for NLR was found to be a cut-off value which can be used in order to distinguish recurrence according to Youden index. According to this, patients with a higher NLR value than 2.494 had higher rates of PSA recurrence with $89.7 \%$ sensitivity and 92.6\% specificity. Conclusions: There are certain parameters used in order to predict recurrence with today's literature data.We think that because NLR is easy to use in clinics and inexpensive, and also has high sensitivity and specificity values, it has the potential to be one of the parameters used in order to predict biochemical recurrence in future.
\end{abstract}

Keywords: NLR - prostate cancer - prostatectomy - PSA - recurrence

Asian Pac J Cancer Prev, 16 (5), 1813-1816

\section{Introduction}

Prostate cancer is the most common malignancy among men (Plonis et al., 2014). The rate of prostate specific antigen (PSA) recurrence after radical prostatectomy, which is the gold standard treatment of localised prostate cancer, is 30\% (Tanaka et al., 2011; Kang et al., 2014). The prediction of pre-operative and post-operative recurrence plays an important role in determination of adjuvant treatment strategies (Naito et al., 2010). While the most important parameter in recurrence prediction was surgical pathology in the past; today, nomograms created with various parameters are used in prediction of post-operative recurrence (Teeter et al., 2013; Kang et al., 2014).

Previous studies have shown that systemic inflammation have an important role in cancer development (Shebl et al., 2014). Similarly, a cancer related inflammatory response occurs in oncologic patients and the severity of this response can be measured with certain indicators in blood (Cetin et al., 2013). One of these indicators is ratio of neutrophil count to lymphocyte count (NLR) in blood. In previous studies, it has been found that increased NLR was associated with poor prognosis and low survival in various cancer types (Sonpavde et al., 2014). In recent years, the relationship between NLR and urological malignancies has been studied and it has been found that NLR was associated with poor prognosis in renal, bladder and castration resistant prostate cancer as well as other cancer types (Cetin et al., 2013; Sonpavde et al., 2014; Hermanns et al., 2014). It is reported that NLR can predict benigh prostate hyperplasia progression (Tanik et al., 2014).

There is a limited number of studies on the relationship between NLR and prostate cancer in the literature (Keizman et al., 2012; Nuhn et al., 2013; Sonpavde et al., 2014; Sumbul et al., 2014). The aim of this study is to research the importance of NLR in prediction of PSA recurrence after radical prostatectomy, which has not been reported so far.

\section{Materials and Methods}

The data of 175 patients, who were diagnosed with localised prostate cancer and underwent retropubic radical prostatectomy in our clinic between 2007 and 2012, was retrospectively examined.Patients with hematological, immunological or thyroid disease and who use steroids and have active infections were excluded from study, 
since their immune responses could be affected. Patients' pre-operative hemogram parameters of neutrophil count, lymphocyte count and NLR were calculated. Patients' ages, pre-operative gleason scores, PSA values, f/tPSA ratios, gleason scores of post-operative pathological specimen, surgical margin positivenesses, perineural invasion presences, seminal vesicle involvements were recorded. The patients whose PSAs were too low to measure after radical prostatectomy in their follow-ups, and then had PSAs of $0,2 \mathrm{ng} / \mathrm{mL}$ were considered as patients with PSA recurrence. Patients with recurrence made up Group A and patients without recurrence made up Group B.

SPSS 15 was used in order to evaluate the obtained data statistically. Firstly, the distributions of the variables on the basis of groups were tested using Kolmogorov Smirnov and Shapiro Wilk tests, and non-parametric test methods were used in the case of absence of a normal distribution. In this context, Mann-Whitney U test was used in order to compare the variables obtained as a result of measurements in two independent groups, and chi-square and/or Fisher exact tests were used in order to examine the differences or the relationship between groups in terms of categorical variables. The areas under the Operating Characteristic Curve (OCC) were used in order to test the distinctivenesses of recurrence and NLR parameters. With this analysis, sensitivity and selectivity of the parameters were calculated as well, and optimal cutting points were determined according to Youden index. Also, Pearson and Spearman correlation coefficients were used in order to examine the relationship between the parameters.

\section{Results}

Among a total of 175 patients, there were 39 patients (22\%) with recurrence in Group A and 136 patients (78\%) without recurrence in Group B. There was a statistically significant difference between the patient ages of Group A and Group B $(p=0,005)$. Median follow-up time was 32(12-68) months for group A and 29(12-90) months for Group B.There wasn't a statistically significant difference between two groups in this respect (Table 1).

While there was surgical margin positiveness in 26 $(66,7 \%)$ out of 39 patients with PSA recurrence, surgical border positiveness couldn't be detected in $13(33,3 \%)$ patients. According to this, there was a statistically significant difference between PSA recurrence detection and surgical margin positiveness $(\mathrm{p}=0,004)$ (Table 1$)$.

There wasn't a statically significant difference between the groups in terms of PSA and f/tPSA ratio, perineural invasion and seminal vesicle invasion presence and PSA recurrence (Table 1).

In terms of pre-operative prostate biopsy gleason score and post-operative specimen Gleason score, there was a statistically significant difference between patients who had the same score, patients who had lower scores compared to their biopsy scores (downgrading), patients who had higher scores compared to their biopsy scores (upgrading) and PSA recurrence detection $(\mathrm{p}<0,001)$. According to this, PSA recurrence was more common in
Table 1. Median (min-max) and Numerical (percentage) Data of the Patients in the Two Groups

\begin{tabular}{lccc}
\hline & Group A & Group B & p value \\
\hline Number of Patients & $39(22 \%)$ & $136(78 \%)$ & \\
Age & $67(59-76)$ & $65(49-75)$ & $0.005^{*}$ \\
PSA (ng/dL) & $7,4(2,2-35)$ & $7,05(1,1-49)$ & 0.23 \\
f/t PSA (\%) & $14(3-48)$ & $13(1-40)$ & 0.822 \\
Follow-up Time (months) & $32(12-68)$ & $29(12-90)$ & 0.144 \\
Surgical Margin Positiveness & & 0.004 \\
$\quad$ No & $13(14 \%)$ & $81(86 \%)$ & \\
$\quad$ Yes & $26(32 \%)$ & $55(68 \%)$ & \\
Perineural Invasion & & & 0.188 \\
$\quad$ No & $25(26 \%)$ & $71(74 \%)$ & \\
$\quad$ Yes & $14(18 \%)$ & $65(82 \%)$ & \\
Seminal Vesicle Invasion & & & 0.4 \\
$\quad$ No & $13(27 \%)$ & $36(73 \%)$ & \\
$\quad$ Yes & $26(20 \%)$ & $100(80 \%)$ & \\
Gleason Score Difference & & & $<0.001 *$ \\
$\quad$ Same & $5(5 \%)$ & $92(95 \%)$ & \\
$\quad$ Low (downgrading) & $2(8 \%)$ & $23(92 \%)$ & \\
$\quad$ High (upgrading) & $32(60 \%)$ & $21(40 \%)$ & \\
\hline
\end{tabular}

Table 2. Median (min-max) and Numerical (percentage) Data of the Patients in Two Groups According to NLR

\begin{tabular}{lccc}
\hline & NLR $\leq 2,494$ & $>2,494$ & $\mathrm{p}$ value \\
\hline Number of Patients & $130(74 \%)$ & $45(26 \%)$ & \\
Age & $65(49-75)$ & $67(55-76)$ & 0.08 \\
PSA (ng/dL) & $7.2(1.1-49)$ & $7.1(2.2-35)$ & 0.271 \\
f/t PSA (\%) & $14(1-40)$ & $12(3-48)$ & 0.66 \\
Follow-up time (months) & $29(12-90)$ & $31(12-68)$ & 0.39 \\
Recurrence & & & $<0.001 *$ \\
$\quad$ No & $126(92,6 \%)$ & $10(7,4 \%)$ & \\
$\quad$ Yes & $4(10,3 \%)$ & $35(89,7 \%)$ & \\
Surgical Margin Positiveness & & 0.013 \\
$\quad$ No & $77(81,9 \%)$ & $17(18,1 \%)$ & \\
$\quad$ Yes & $53(65,4 \%)$ & $28(34,6 \%)$ & \\
Perineural Invasion & & & 0.065 \\
$\quad$ No & $66(68,8)$ & $30(31,2 \%)$ & \\
$\quad$ Yes & $64(81)$ & $15(19 \%)$ & \\
Seminal Vesicle Invasion & & & 0.878 \\
$\quad$ No & $36(73,5 \%)$ & $13(26,5 \%)$ & \\
$\quad$ Yes & $94(74,6 \%)$ & $32(25,4 \%)$ & \\
Gleason Score Difference & & & $<0.001 *$ \\
$\quad$ Same & $88(90 \%)$ & $9(10 \%)$ & \\
$\quad$ Low & $22(88 \%)$ & $3(12 \%)$ & \\
$\quad$ High & $20(38 \%)$ & $33(62 \%)$ & \\
\hline
\end{tabular}

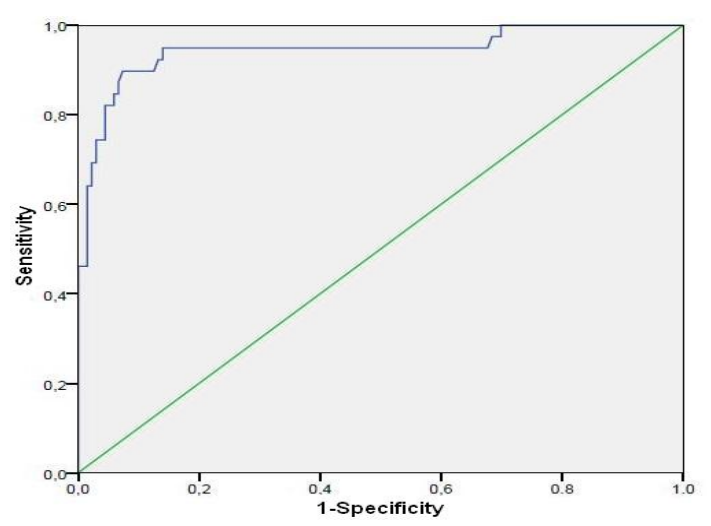

Figure 1. Operation Characteristic Curve (OCC)

upgrading group (Table 1).

In terms of the power of NLR value in distinguishing 
recurrence, the area under OCC was statistically significant $(\mathrm{p}<0,001)$ (Table 2). The value of 2,494 for NLR was found to be a cut-off value which can be used in order to distinguish recurrence according to Youden index. According to this, patients with a higher NLR value than 2,494 had higher rates of PSA recurrence with $89,7 \%$ sensitivity and $92,6 \%$ specificity (Figure 1).

There wasn't a statistically significant difference in terms of PSA, f/tPSA, surgical margin positiveness, seminal vesicle involvement and perineural invasion presence between patients below and above the NLR cutoff value. In terms of pre-operative prostate biopsy gleason score and post-operative prostate specimen gleason score, there was a statistically significant difference between patients who had the same score, patients who had lower scores compared to their biopsy scores (downgrading), patients who had higher scores compared to their biopsy scores (upgrading) and patients below and above the NLR cut-off value $(\mathrm{p}<0,001)$. According to this, operative pathology Gleason scores of patients with NLR values higher than 2,494 were higher (Table 2).

\section{Discussion}

The effect of inflammation and immune response against inflammation on carcinogenesis has been shown in various studies (Shebl et al., 2014). The detection of immune response against tumor with certain markers is quite easy in clinical practice. NLR is one of the markers that shows immune response (Sonpavde et al., 2014). The fact that NLR can be easily and inexpensively measured with hemogram parameters and increased NLR is a poor prognosis factor in many types of cancer has made NLR popular among clinicians.

NLR's relationship with poor prognosis was first found with gastric cancers and then studies on colorectal hepatocellular and ovary cancers have been conducted. Increased levels of NLR have been found to be a poor prognosis factor in all these cancer types (Eerola et al., 2006; Kishi et al., 2009; Ozyalvaclı et al., 2014). NLR's relationship with poor prognosis can be explained with several mechanisms. Immune response of patient is lymphocyte mediated. Patients with high NLRs have relatively low lymphocyte counts and sufficient immune effect against tumor can not be developed in these patients. On the other hand, high neutrophil counts in blood causes an increase in vascular endothelial growth factor (VEGF) emission which plays an important role in tumor progression and angiogenesis. Accordingly; increased tumor recurrence, poor prognosis and decreased survival were seen (Kusumanto et al., 2003; Fondevila et al., 2004)

For this reason, similar studies have been conducted on NLR in urological malignancies in recent years (Wei et al., 2014). It has been found that neutrophilia and lymphocytopenia were associated with poor prognosis in metastatic renal tumors (Ohno et al., 2010). Ohno et al. have found that among patients with metastatic renal tumors, patients with NLR values with lower than 2,7 had 5-year and 10 year recurrence-free survivals of $93,7 \%$ and $79,8 \%$ respectively, patients with NLR values with higher than 2,7 had 5-year and 10 year recurrence-free survivals of $77,9 \%$ and $58,4 \%$ respectively $(p=0,02)$. In the same study, it has been emphasised that NLR was an independent predictive parameter for recurrence (Ohno et al., 2010).

Ceylan et al. have found that NLR value of patients with muscle invasive bladder tumors was $4,14 \pm 2,76$ and NLR value of patients with non-muscle invasive bladder tumors was $3,36 \pm 2,88$, and that this difference was statistically significant ( $\mathrm{p}=0,03)$ (Ceylan et al., 2014). In a study on patients who underwent radical cystectomy due to invasive bladder tumor, NLR cut-off value was found to be 3,0 and patients with NLR values higher than this had a significantly lower recurrence-free survival (Hermanns et al., 2014)

There is a limited number of studies on the relationship between NLR and prostate cancer in the literature (Keizman et al., 2012; Nuhn et al., 2013; Sonpavde et al., 2014; Sumbul et al., 2014). In a study conducted with the participation of 238 patients with castration resistant metastatic prostate cancer, patients with pre-docetaxel treatment NLR values lower than 3 responded better to treatment and had significantly higher total survival compared to patients with NLR values higher than 3 (Nuhn et al., 2013). In another study conducted with the same patient group, the patients have been divided into two groups as those with pre-docetaxel treatment NLR values lower than 3 and those with pre-docetaxel treatment NLR values higher than 3, while there wasn't a significant difference between the groups in terms of total survival, patients with pre-docetaxel treatment NLR values lower than 3 had a significantly high decrease in post-operative PSA (Sumbul et al., 2014).

Patients who received definitive treatment due to prostate cancer have considerably high recurrence rates. $27-53 \%$ of patients who underwent radical prostatectomy or received radiotherapy treatment as primary treatment had local or systemic recurrence in 10 years, and 16-35\% of these patients needed secondary treatment in 5 years (Kang, 2014).

PSA decrease to an unmeasurable level or to rare value and then starting to increase is referred to as biochemical recurrence. Lange et al. has shown that biochemical recurrence predicted systemic recurrence 6-48 months in advance. In about half of patients with PSA recurrence, disease shows progression and it is known that secondary treatments provide an advantage in terms of survival in this patient group (Fichtner et al., 2000).

Post-operative biochemical recurrence rate in prostate cancer is approximately $30 \%$ (Kang, 2014). This patient group with recurrence should be followed closely and receive adjuvant treatments if necessary (Ploussard et al., 2011). Post-operative PSA recurrence depends on surgical margin positiveness, extraprostatic extension of tumor, seminal vesicle involvement, pathological specimen gleason score and pre-operative PSA value of the patient (Ploussard et al., 2011). According to studies; in recurrence prediction, the most important parameter in progression and survey of disease is surgical margin positivenesses (Ploussard et al., 2011; De La Roca et al., 2014). Examining the literature data, surgical margin positivenesses was approximately $40 \%$ and PSA 
recurrence ratio was $29.8 \%$ of these patients (De La Roca et al., 2014). Although follow-up lengths were short in our study, while surgical margin positivenesses was $46 \%, 32 \%$ of these patients developed PSA recurrence. Also, patients with a higher surgical specimen gleason score compared to their biopsy Gleason scores had a significantly higher recurrence rate $(\mathrm{p}<0,001)$.

In conclusion, the prediction of post-operative recurrence plays an important role in determination of adjuvant treatment strategies and patient follow-up (Cetin et al., 2013; Kang, 2014). There are certain parameters used in order to predict recurrence with today's literature data (Kang, 2014). We think that because NLR is easy to use in clinics and inexpensive, and also has high sensitivity and specificity values, it has the potential to be one of the parameters used in order to predict biochemical recurrence in future..

\section{References}

Cetin B, Berk V, Kaplan MA,, et al (2013). Is the pretreatment neutrophil to lymphocyte ratio an important prognostic parameter in patients with metastaticrenal cell carcinoma? Clin Genitourin Cancer, 11, 141-8.

Ceylan C, Doluoglu OG, Keles I,et al (2014). Importance of the neutrophil-to-lymphocyte ratio in muscle-invasive and non-muscle invasive bladder tumors. Urologia, 81, 120-4.

De La Roca RL, Da Cunha IW, Bezerra SM, Da Fonseca FP (2014). Radical prostatectomy and positive surgical margins: relationship with prostate cancer outcome. Int Braz J Urol, 40, 306-15.

Eerola AK, Soini Y, Paakko P (2006). A high number of tumorinfiltrating lymphocytes are associated with a small tumor size, low tumor stage and a favorable prognosis in operated small cell lung carcinoma. Clin Cancer Res, 6, 1875-81.

Fichtner J (2000). The management of prostate cancer in patients with a rising prostate-specific antigen level. BJU Int, 86, 181-90.

Fondevila C, Metges JP, Fuster J et al(2004). P53 and VEGF expression are independent predictors of tumour recurrence and survival following curative resection of gastric cancer. Br J Cancer, 90, 206-15.

Hermanns T, Bhindi B, Wei Y, et al (2014). Pre-treatment neutrophil-to-lymphocyte ratio as predictor of adverse outcomes in patients undergoing radicalcystectomy for urothelial carcinoma of the bladder. Br J Cancer, 29, 444-51.

Kang M, Jeong CW, Choi W, et al (2014). Pre- and post-operative nomograms to predict recurrence-free probability in korean men with clinically localized prostate cancer. PLoS One, 17, 100053 .

Keizman D, Gottfried M, Ish-Shalom M, et al (2012). Pretreatment neutrophil-to-lymphocyte ratio in metastatic castration-resistant prostate cancer patients treated with ketoconazole: association with outcome and predictive nomogram. Oncologist, 17, 1508-14.

Kishi Y, Kopetz S, Chun YS, et al (2009). Blood neutrophil-tolymphocyte ratio predicts survival in patients with colorectal liver metastases treated with systemic chemotherapy. Ann Surg Oncol, 16, 614-22.

Kusumanto YH, Dam WA, Hospers GA, Meijer C, Mulder NH (2003). Platelets and granulocytes, in particular the neutrophils, form important compartments for circulating vascular endothelial growth factor. Angiogenesis, 6, 283-87

Naito S (2010). Evaluation and management of prostate-specific antigenrecurrence after radical prostatectomy for localized prostate cancer. J Clin Oncol, 35, 365-74.

Nuhn P, Vaghasia AM, Goyal J, et al (2013). Association of pretreatment neutrophil-to-lymphocyte ratio (NLR) and overall survival (OS) in patients withmetastatic castrationresistant prostate cancer (mCRPC) treated with first-line docetaxel. BJU Int.

Ohno Y, Nakashima J, Ohori M, Hatano T, Tachibana M (2010). Pretreatment neutrophil-to-lymphocyte ratio as an independent predictor of recurrence in patients with nonmetastatic renal cell carcinoma. J Urol, 184, 873-78.

Ozyalvacli G, Yesil C, Kargi E, et al (2014).Diagnostic and prognostic importance of the neutrophil lymphocyte ratio in breast cancer. Asian Pac J Cancer Prev, 15, 10363-6.

Plonis J, Bokums K, Cauce V, et al (2014). Prostate cancer trends in Latvia during 1990-2012: Incidence, prevalence, mortality, and survival rates. Medicina, 50, 313-7.

Ploussard G, Agamy MA, Alenda O,, et al (2011). Impact of positive surgical margins on prostate-specific antigen failure after radicalprostatectomy in adjuvant treatment-naïve patients. BJU Int, 107, 1748-54.

Shebl FM, Hsing AW, Park Y, et al (2014). Non-steroidal antiinflammatory drugs use is associated with reduced risk of inflammation-associated cancers: NIH-AARP study. PLoS One, 9, 114633.

Sonpavde G, Pond GR, Armstrong AJ, et al (2014). Prognostic impact of the neutrophil-to-lymphocyte ratio in men with metastatic castration-resistant prostate cancer. Clin Genitourin Cancer, 14, 54-8.

Sumbul AT, Sezer A, Abalı H, et al (2014). Neutrophil-to lymphocyte ratio predicts PSA response, but not outcomes in patients with castration resistantprostate cancer treated with docetaxel. Int Urol Nephrol, 46, 1531-5.

Tanaka N, Fujimoto K, Hirayama A, et al (2011). Risk-stratified survival rates and predictors of biochemical recurrence after radical prostatectomy in a Nara, Japan, cohort study. Int $J$ Clin Oncol, 16, 553-9.

Tanik S, Albayrak S, Zengin K, et al (2014). Is the neutrophillymphocyte ratio an indicator of progression in patients with benign prostatic hyperplasia? Asian Pac J Cancer Prev, 15, 6375-9.

Teeter AE, Presti JC Jr, Aronson WJ, et al (2013). Do nomograms designed to predict biochemical recurrence (BCR) do a better job of predicting more clinically relevant prostate cancer outcomes than BCR? A report from the SEARCH database group. Urology, 82, 53-8.

Wei Y, Jiang YZ, Qian WH (2014). Prognostic role of NLR in urinary cancers: a meta-analysis. PLoS One, 18, 92079. 